
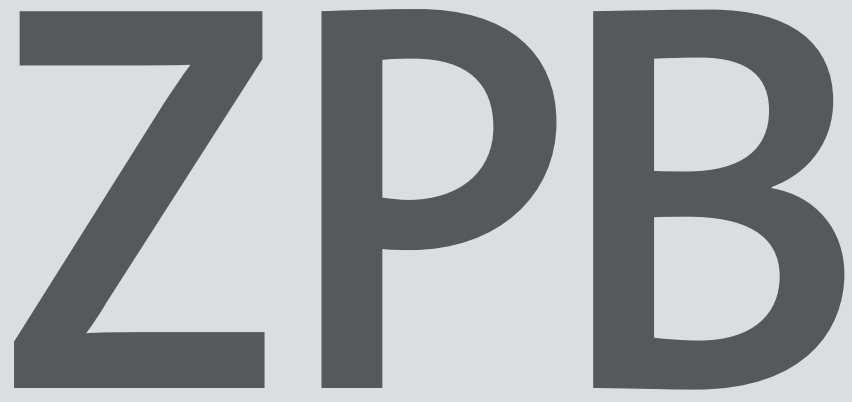

\title{
$2_{\mid 2013}$ \\ 6. Jahrgang, Seiten $53-108$ Zeitschrift für
}

\section{POLICY ADVICE AND POLITICAL CONSULTING}

Herausgeber/innen: Prof. Dr. Andrea Römmele, Hertie School of Governance, Berlin (Geschäftsführende Herausgeberin), E-Mail: roemmele@hertie-school.org | Dr. Svenja Falk, Accenture Research, Berlin, Germany, E-Mail: svenja.falk@accenture.com | PD Dr. Martin Thunert, Center for American Studies, Universität Heidelberg, E-Mail: mthunert@hca.uni-heidelberg.de

Beirat: Prof. Dr. h.c. Roland Berger, Roland Berger Strategy Consultants | Vito Cecere, Auswärtiges Amt | Prof. Dr. Jobst Fiedler, Hertie School of Governance | Prof. Dr. Dietmar Fink, Hochschule Bonn-Rhein-Sieg | Prof. Dr. Justus Haucap, Heinrich-Heine-Universität Düsseldorf | Dr. Christian Humborg, Transparency International Deutschland e.V.| Nikolaus Huss, Politik Kommunikation | Prof. Dr. Dr. Karl-Rudolf Korte, Universität Duisburg-Essen | Prof. Dr. Sabine Kropp, Deutsche Hochschule für Verwaltungswissenschaften Speyer | Prof. Dr. Martin Lendi, Eidgenössische Technische Hochschule Zürich | Prof. Dr. Miriam Meckel, Universität St. Gallen | Prof. Dr. Ferdinand Müller-Rommel, Leuphana Universität Lüneburg | Dr. Leonard Novy, Institut für Medien- und Kommunikationspolitik | Cem Özdemir, BÜNDNIS 90/DIE GRÜNEN | Anke Plättner, Westdeutscher Rundfunk | Peter Radunski, MSL Group | PD Dr. Dieter Rehfeld, FH Gelsenkirchen | Prof. Volker Riegger, logos Holding AG | Prof. Dr. Stefan A. Schirm, Ruhr-Universität Bochum | Dr. Rolf Steltemeier, Bundesministerium für wirtschaftliche Zusammenarbeit und Entwicklung | Cornelius Winter, de'ge'pol

Redaktion: Henrik Schober, M.A. (Chefredakteur) | Johannes Staemmler, MPP | Andreas Hoffelder, Dipl. Sozwiss.

Kontakt: Zeitschrift für Politikberatung (ZPB), c/o Henrik Schober, M.A., Hertie School of Governance, Quartier 110, Friedrichstraße 180, 10117 Berlin, Telefon: +49 - 30 / 259219 338, E-Mail: schober@hertie-school.org

\section{Verwaltungsmodernisierung in Sachsen}

\section{Modellpräferenz, Reformpfad, Aussichten und Hemmnisse}

Markus Reiners

\section{Kernaussagen}

Sachsen modernisiert seine Verwaltungsstrukturen. Dazu fragt dieser Beitrag, worauf sich die jüngsten Strukturentscheidungen zurückführen lassen bzw. wie die Handlungspfade im Freistaat ausgestaltet sind. Der vormalige Verwaltungsaufbau erinnert noch an den Strukturimport aus Baden-Württemberg Anfang der 1990er Jahre. Bei diesem standen noch dezentrale Strukturen im Vordergrund. Nach einer Zeit der Konsolidierung und aufgrund zwischenzeitlich veränderter finanzieller Rahmenbedingungen sowie aufgrund der fortschreitenden Europäisierung verfolgt Sachsen nunmehr den Weg einer strukturellen Zentralisierung und orientiert sich hierbei vorwiegend an den beiden Nachbarländern Sachsen-Anhalt und Thüringen, die beide eine staatliche Mittelinstanz mit nur einer Bündelungsbehörde vorhalten. Da diese Struktur nur in diesen drei Ländern vorzufinden ist, kann auch von einem dreigliedrigen ostdeutschen Modell gesprochen werden.

\section{Strukturwandel und Forschungsfrage}

Sachsen modernisiert seine Verwaltungsstrukturen. Die entscheidende Frage lautet hierbei, worauf sich die jüngsten Strukturentscheidungen - mit Blick auf die staatliche Mittelebene - zurückführen lassen. Wie also sind die Handlungspfade im Freistaat ausgestaltet, sodass es zu ganz bestimmten und nicht etwa anderslautenden Richtungsweisungen kommt? Warum wird ein ganz bestimmtes Verwaltungsmodell präferiert? Ebenso darf die begleitende Frage lauten, ob damit die Weichen richtig gestellt sind und das Vorhaben durchsetzbar erscheint. Die strukturpolitischen Entscheidungen hängen sicherlich von einem umfassenden Kriterienkatalog ab und sind in der hier gebotenen Kürze allenfalls vage zu prognostizieren. Letztlich hängen diese auch von temporären Faktoren ab, denn die Reform muss sich im Laufe der Zeit erst beweisen.

Blickt man insgesamt auf die Aktivitäten der Bundesländer hinsichtlich ihrer Verwaltungsstrukturen, so lassen sich unter dem Diktat schwindender Ressourcen seit den 1990er Jahren verstärkt Reformbemühungen feststellen. Seit der deutschen Wiedervereinigung zwingen die gestiegenen Haushaltsdefizite das System dazu, sich zunehmend selbst in den Blick zu neh- 
men und sein organisatorisches Gefüge zum Gegenstand der Politik zu machen. Finanzielle Restriktionen haben sich hierbei fast durchweg als maßgeblich erwiesen, wobei weitere veränderte Rahmenbedingungen hinzutreten, die beispielsweise von einer fortschreitenden Europäisierung und Globalisierung über wachsende territoriale und soziale Disparitäten bis hin zu den Konsequenzen der demografischen Entwicklung reichen (Luhmann, 1984; Lüder 2004, S. 76; Hesse, 2010, S. 12-13; Hesse, 2011 a, S. 16-17).

Neben Einspareffekten geht es entscheidend um eine inhaltlich funktionale Neuausrichtung des öffentlichen Bereichs, sowohl vertikal als auch horizontal. Die Reformansätze zielen auf eine Vereinfachung der Staatsorganisation durch Auflösung, Zusammenlegung und Ausgliederung von Einrichtungen. Die Verwaltung soll mit Blick auf die eigene Organisation effektiver, effizienter, bürgernäher und kritischer arbeiten. Vordergründig geht es vielfach um eine Entflechtung und Verantwortungsteilung, eine klare Aufgabenzuordnung, eine Reduzierung und Konzentration von Aufsichtstätigkeiten, eine Verantwortungsdelegation sowie eine Integration von Koordinationssystemen, was die Frage einer sinnvollen Arbeitsaufteilung zwischen Land und Kommunen berührt. Die Ausführungen plausibilisieren, dass eine zeitgemäße und nachhaltige Verwaltungsreform nicht singulär auf die Landesebene oder die kommunale Stufe bezogen sein kann, sondern beide Ebenen in einem untrennbaren Interaktionsverhältnis stehen. Insgesamt ist in den Ländern eine hohe Reformbereitschaft zu diagnostizieren. Die Maßnahmen reichen von punktuellen Anpassungen bis hin zu einem Systemwechsel. Im Mittelpunkt der Diskussion stehen hierbei vorwiegend Modelle einer Zwei- und einer konzentrierten Dreigliedrigkeit (Freudenberg, 2000, S. 39; Bouckaert, 2004, S. 22; Reiners, 2008, S. 25, 71-78; Hesse, 2010, S. 17-20, 40; Hesse, 2011 a, S. 18-22; Jacob, 2011 a).

Der Reformeifer hat schon länger auch die ostdeutschen Länder erfasst. Die Länder Brandenburg und MecklenburgVorpommern haben auf Mittelbehörden verzichtet, doch finden sich solche in Sachsen, Sachsen-Anhalt und Thüringen, die dreistufig organisiert sind. In Sachsen-Anhalt wurden jedoch mit Wirkung zum 1. Januar 2004 die drei Regierungspräsidien zu Gunsten eines zentralen Landesverwaltungsamtes aufgegeben. Wie in Thüringen fungiert dort ein solches als Bündelungsbehörde. Sachsen war bisher das einzige neue Bundesland mit noch drei Mittelbehörden. Nach der Wiedervereinigung orientierten sich die neuen Länder zunächst an ihren westdeutschen Vorbildern (Häusler, 2004, S. 147; Seitz, 2007; Hesse, 2010, S. 19; Hesse, 2011 a, S. 20-21; Siedentopf \& Laux, 1998). Im Laufe der Jahre hat jedoch nicht nur der Freistaat zu einer größeren Eigenständigkeit gefunden, die ein oder andere Funktionalreform durchgeführt und die Strukturen seinen Bedürfnissen und strukturellen Rahmenbedingungen entsprechend - sukzessive angepasst. Die drei ehemaligen Regierungspräsidien wurden daher im Jahr 2008 in sogenannte Landesdirektionen umbenannt. In diesem Kontext ist die bislang nicht evaluierte Funktionalreform des Jahres 2008 von Belang. Hierbei kam es zu einer umfassenden Aufgabenkommunalisierung, zu einer nachhaltigen Stärkung des kommunalen Bereichs und zu einer Zentralisierung auf der unteren Stufe, indem die 22 Landkreise auf zehn plus die drei kreisfreien Städte Chemnitz, Dresden und Leipzig reduziert wurden.

Aufgrund geänderter Rahmenbedingungen geht es in Sachsen heute weniger um den Aspekt der Dezentralisierung, als Maxime der 1990er Jahre, sondern - nach einer strukturellen Konsolidierung - mehr um Zentralisierungseffekte und entscheidend um die Schaffung zukunftsträchtiger Strukturen als Baustein der Staatsmodernisierung und letztlich um die Reduzierung der drei staatlichen Mittelbehörden auf eine Landesdirektion: „In den ersten zehn Jahren war es gut, dass wir beim Aufbau (...) dezentrale Entscheidungsstrukturen hatten. Dann hatten wir im zweiten Jahrzehnt (...) eher eine Konsolidierung der Strukturen. Wir haben jetzt im Grunde genommen die Anpassung an die Zukunft“ (Jacob, 2011 a, S. 54).

\section{Theoretische Einordnung}

Schaut man auf die aktuellen Vorgänge so ist vorab festzustellen, dass sich diese neoinstitutionalistisch erfassen lassen. Die Studie lehnt sich daher zunächst an den historischen Institutionalismus an (Steinmo, Thelen \& Longstreth, 1992; Thelen, 2002; Evans, Rueschemeyer \& Skocpol, 1985; Hall \& Taylor, 1996; Kaiser, 1999, S. 191). Dieser betont als Erklärungsstand Institutionen und die davon ausgehenden Pfadabhängigkeiten. Fragt man sich nach den Maßgeblichkeiten, die als Entscheidungsgrundlagen sekundieren, so wird deutlich, dass die etablierten Modernisierungspfade anderer Länder eine Rolle spielen. So orientiert sich Sachsen an Ländern, die eine Dreigliedrigkeit präferieren. Entscheidend sind möglicherweise auch die Pfade der beiden kleineren Nachbarländer Sachsen-Anhalt und Thüringen. Weiterhin ist der akteurszentrierte Institutionalismus für die Untersuchung bedeutsam (Mayntz \& Scharpf, 1995; Scharpf, 2000; Benz, 2001, S. 76). Dieser betont, dass die institutionellen Bedingungen die Handlungspräferenzen formen, diese allerdings nicht exogen vorgegeben sind. Vielmehr prägt das Umfeld die Ziele und die Wahl der Mittel zur Zielerreichung ohne Determination, denn zwischen Institution und Handlung liegt die Wahrnehmung und Interpretation der Akteure. Im Mittelpunkt stehen damit Erklärungen des Zusammenwirkens von Institutionen, Interaktionen, Akteurshandeln und Politikergebnissen. Insgesamt ist die Prägekraft institutioneller Faktoren maßgeblich, die einen ermöglichenden oder restringierenden Kontext bilden und damit auf die Verläufe und Ergebnisse wirken.

Die Ansätze, als Erklärungsinstrumente der Reformierbarkeit politischer Systeme und Strukturen, können noch präzisiert werden, wenn sie mit dem Vetospielerkonzept verbunden werden. Grundlegend sind hierbei die Hypothesen von Tsebelis, der aus der Existenz von Vetospielern bzw. aus ihren durch interne Strukturen vermeintlich vorgegebenen Handlungsweisen auf Ergebnisse schließt (1995; 2002; Kaiser, 1998; Bandelow, 2003 a; Bandelow, 2003 b; Benz, 2003, S. 208-209). 
Für die vorliegende Analyse ist dies insofern erweiterungsbedürftig, weil unter bestimmten Voraussetzungen potenzielle Vetokräfte Veränderungen nicht nur destruktiv blockieren sondern solche auch begünstigen können (Bandelow, 2003 a). Benz folgert deshalb, dass es entscheidend ist den strategischen Umgang der Akteure mit Vetomacht bzw. das Zusammenspiel von Handlungsbedingungen und -strategien zu berücksichtigen, was es erlaubt den Ansatz unter neoinstitutionalistische Konzepte zu rubrizieren. Dabei stellt sich die Frage, unter welchen Bedingungen potenzielle Vetospieler eine konstruktive Politik betreiben und wann nicht (2003, S. 208-209; Nemitz, 2000).

\section{Verwaltungsmodernisierung in Sachsen}

\subsection{Zielperspektiven und Reforminhalte}

Im Koalitionsvertrag zwischen CDU und FDP zur fünften Legislatur wurde das Vorhaben einer grundlegenden Modernisierung vereinbart (Sächsische Staatsregierung, 2011). Das Gesetz zur Neuordnung von Standorten der Verwaltung und der Justiz des Freistaates Sachsen (Sächsisches Standortegesetz - SächsStOG) wurde in der weiteren Folge vom Sächsischen Landtag am 25. Januar 2012 verabschiedet und ist zum 1. März 2012 in Kraft getreten. Damit soll bis 2020 das Ziel einer dauerhaft leistungsfähigen Verwaltung, angepasst an die sich ändernden finanziellen und bevölkerungspolitischen Rahmenbedingungen, verfolgt werden. Da das Vorhaben in Abhängigkeit zu anderen Konzeptionen steht, können die Einzelmaßnahmen lediglich nach und nach und in zeitlich abgestimmter Folge durchgeführt werden. Sie stehen unter dem Vorbehalt, dass hierfür die haushaltsrechtlichen Voraussetzungen geschaffen werden.

Als dominantes Ziel stehen finanzielle Einsparungen im Vordergrund. Die sozio-ökonomische Ausgangslage hat einen vielfach stimulierenden Charakter, um eine umfassende Reform überhaupt anzugehen und einen Paradigmenwechsel einzuleiten (Beyer, 2011; Reiners, 2008, S. 196). Plausibel werden die Ausführungen, wenn man einen relativen Indikator anlegt, die Pro-Kopf-Verschuldung. Zwar schneidet der Freistaat mit Blick auf andere Länder vergleichsweise gut ab, denn Sachsen liegt mit der niedrigsten Pro-Kopf-Verschuldung bundesweit an der Spitze, jedoch wird der finanzielle Handlungsrahmen überall nach und nach geringer (Wirtschaftswoche, 20.10.2011). Daher erscheint eine perspektivische Anpassung der Strukturen, verknüpft mit einem Personalabbau mit Augenmaß, angezeigt und sinnvoll.

Die Regierung hat für den Umsetzungszeitraum bis 2021 insgesamt einen Betrag von 295 Mio. $€$ ermittelt. Dem steht durch die Umsetzung der Stellenabbauverpflichtung von ca. 17.000 Stellen in den nächsten zehn Jahren und weiterer Einsparungen, z.B. durch Veräußerungserlöse und entfallende Mietaufwendungen, insgesamt ein Einsparvolumen von rund 1 Mrd. $€$ gegenüber, sodass sich per Saldo ein finanzieller Vorteil von über 842 Mio. $€$ ergeben soll. Nach der Umsetzung ab 2022 stehen den erwarteten jährlichen Mehrkosten von $146.000 €$ jährliche Einsparungen von ca. 285 Mio. $€$ an Personal-, Sach- und Mietkosten gegenüber, so die Regierung (Beyer, 2011; Sächsische Staatsregierung, Vorblatt, S. 2; Wirtschaftswoche, 20.10.2011). Fraglich ist hierbei, ob sich der finanzielle Vorteil derart exakt berechnen lässt. Lässt sich eine kurzfristige Kalkulation vielfach noch relativ valide gestalten, so wird eine solche sicherlich mit jedem weiteren Jahr unverlässlicher. Wie passen die Sachverhalte z.B. zu künftigen Finanz- und Wirtschaftskrisen, wie stabil bleibt unsere Währung, welche demografischen Gesichtspunkte stehen mit der Reform in Konflikt, wie wirken sich Lebensarbeitszeitverlängerungen der Mitarbeiterschaft auf die Berechnungen aus, alles Fragen von hoher Unsicherheit (Schreiter, 2011).

Als weiteres wichtiges Ziel steht ein verändertes gesellschaftliches Kommunikationsverhalten im Fokus. Die Entwicklung neuer Informationstechniken hat zwischenzeitlich zu starken Veränderungen geführt. Das Internet ist fester Bestandteil unserer Gesellschaft und mittlerweile hat jeder zweite deutsche Internetnutzer Electronic-Government-Angebote in Anspruch genommen (Lucke \& Reinermann, 2000; Gisler, 2001; ZEIT Online, 27.5.2004). Durch die elektronische Modernisierung sollen Kosten gesenkt und die Attraktivität des Standorts gesteigert werden. Des Weiteren soll mehr Bürgernähe geschaffen und die Kommunikation mit und zwischen Behörden erleichtert werden, was künftig einen schlankeren Verwaltungsaufbau nahelegt.

Hohe Relevanz hat das Thema, weil in den vergangenen Jahren eine rasche Weiterentwicklung stattgefunden hat. So befinden wir uns zwischenzeitlich in der Reifephase der dritten Generation eines eGovernment (Grabow, Siegfried \& Wulff, 2006). Während man sich zunächst darauf beschränkte auf den Internetseiten Informationen bereitzustellen, sind inzwischen immer mehr Behörden dazu übergegangen, mit ihrer Bürgerschaft $\mathrm{zu}$ interagieren. Ein weiterer Schritt besteht in der Prozessabwicklung und in Transaktionen. Ist diese Stufe erreicht, sollen die Einzellösungen zu einem konsolidierten Gesamtkonzept verschmelzen. Gekennzeichnet ist diese Phase in erster Linie nicht mehr durch ein verstärktes Angebot, sondern vor allem durch einen Integrationsmechanismus auf diversen Ebenen (Scheer, Heib \& Kruppke, 2003; Grabow et al., 2006, S. 9).

Entscheidend ist in diesem Kontext, dass ein e-Government immer auch moderne Folgeprojekte nach sich zieht und damit als Treiber von Verwaltungsprozessen an Relevanz gewinnt, was insbesondere der Fall ist, wenn ein Zusammenwirken von Informationstechnologie und Organisationsstrukturen erreicht wird. E-Government schafft somit die Voraussetzungen und Handlungspfade für künftige Projekte. Von Bedeutung ist dabei, dass die Einführung eines e-Government zwingend eine Anpassung der Organisationsstrukturen verlangt und die Institutionalisierung einer nachhaltigen Prozesskultur als grundlegende Bedingung erfordert (Reiners, 2011).

Blickt man auf die elementaren Inhalte der Reform, so wird eine Konzentration sowohl auf die untere als auch auf die mittlere Verwaltungsstufe deutlich (Sächsische Staatsregie- 
rung, 2001, Vorblatt, S. 1, Übersicht, S. 5). Neben verschiedenen Maßnahmen aus dem Gesetz - u.a. den Rechnungshof, die Sächsische Aufbaubank-Förderbank, die Landesstiftung Natur und Umwelt sowie die Amts- und Landgerichte betreffend - interessieren hier insbesondere die strukturellen Veränderungen auf der mittleren staatlichen Ebene. Hierzu wurden die drei Landesdirektionen in Chemnitz, Dresden und Leipzig zur Landesdirektion Sachsen mit Hauptsitz in Chemnitz zusammengefasst. Eine Dreistufigkeit bleibt daher auch künftig die Maxime. Die Landesdirektion ist als allgemeine Staatsbehörde dem Staatsministerium des Innern unmittelbar nachgeordnet. Sie ist als höhere Verwaltungsbehörde im Sinne bundesrechtlicher Vorschriften ausgewiesen und nimmt Aufgaben aus mehreren Staatsministerien wahr. Weiterhin ist sie zur Koordination staatlicher Verwaltungstätigkeiten erforderlich. Auf sie gingen mit Inkrafttreten des Gesetzes die Aufgaben und Befugnisse der bisherigen Landesdirektionen über.

Das Gesetz bildet neben diesen Vorgaben ferner die Grundlage für weitergehende Maßnahmen und Standortentscheidungen. Hierzu gehören beispielsweise die Reduzierung der sieben auf fünf Polizeidirektionen und der 72 Polizeireviere auf 41 Revierstandorte, die Zurückführung der 27 Finanzämter auf 16 Finanzämter in den Landkreisen und kreisfreien Städten sowie die Errichtung eines Sonderfinanzamtes, die Fusion des Autobahnamtes und der Straßenbauämter zum Landesamt für Straßenbau und Verkehr, die Straffung der Standorte der Amtsgerichte oder die Errichtung einer gemeinsamen Justizvollzugsanstalt zusammen mit dem Freistaat Thüringen sowie die Schließung zweier Justizvollzugsanstalten. In diesem Zusammenhang kommt es somit - neben der erklärten Reduktion auf der mittleren Stufe - insbesondere zu Reduzierungen, Fusionen oder Straffungen auf der unteren Verwaltungsebene.

\subsection{Verwaltungsmodelle, struktureller Kontext und Reformpfade}

Nachdem viele westdeutsche Länder ihre Verwaltungsstrukturen inzwischen reformiert haben, setzt der Freistaat diesen Trend fort. Zur Beantwortung der Forschungsfrage ist kursorisch auch auf die Verwaltungsmodelle anderer bundesdeutscher Flächenländer zu blicken. Insgesamt hat sich aus Koordinations- und Steuerungsgesichtspunkten in größeren Ländern eine konzentriert dreistufige in kleineren hingegen eine zweistufige Verwaltung durchgesetzt, wobei einschränkend zu betonen ist, dass es sich hier nur um eine Vereinfachung handelt, weil spezifische Sonderformen existieren. Wird im weiteren Fortgang die Größe eines Landes thematisiert, so ist insbesondere auf die Bevölkerungsanzahl abzuheben, denn nach verwaltungswissenschaftlichen Erkenntnissen ist der Bevölkerungsstärke hinsichtlich der Entscheidung für eine bestimmte Verwaltungsgliedrigkeit eine hohe Bedeutung beizumessen, da mit der Einwohnerzahl der Umfang an öffentlich bereitzustellenden Gütern und zu erbringenden Leistungen wächst (Becker, 1989; Reiners, 2008, S. 25-26). Tabelle 1 zeigt die
Flächenländer, sortiert nach der Einwohnerzahl und versehen mit deren Verwaltungsstufigkeit:

\begin{tabular}{|l|c|c|c|c|c|c|c|c|c|c|c|c|c|}
\hline & NW & BY & BW & NDS & HE & SN & RP & SH & BB & SA & TH & MV & Saar \\
\hline EW & 17,8 & 12,6 & 10,8 & 7,9 & 6,1 & 4,1 & 4,0 & 2,8 & 2,5 & 2,3 & 2,2 & 1,6 & 1,0 \\
\hline VS & 3 & 3 & 3 & 2 & 3 & 3 & 3 & 2 & 2 & 3 & 3 & 2 & 2 \\
\hline
\end{tabular}

Tabelle 1: Einwohnerzahl (EW) und Verwaltungsstufigkeit (VS) der Bundesländer (Statistisches Bundesamt: Einwohnerzahlen, Stand 31.12.2011).

Während die größeren westlichen Länder Nordrhein-Westfalen, Bayern, Baden-Württemberg, Hessen und RheinlandPfalz dreistufig organisiert sind, gestalten sich die kleineren wie das Saarland oder Schleswig-Holstein - zweistufig. Seit 2005 ist Niedersachsen, das viertgrößte Flächenland, jedoch auch zweigliedrig aufgebaut und damit Vorreiter unter den großen Ländern. Im östlichen Teil der Republik sind die Länder Brandenburg und Mecklenburg-Vorpommern zweistufig organisiert, die Länder Thüringen und Sachsen-Anhalt dreistufig, beide zwar größer als Mecklenburg-Vorpommern, jedoch kleiner als Brandenburg. Das größte östliche Flächenland Sachsen, von der Größe etwa vergleichbar mit RheinlandPfalz, präferiert auch künftig eine Dreigliedrigkeit, obwohl die Prognose sinkende Bevölkerungszahlen andeutet und im Jahre 2020 vermutlich noch knapp 3,9 Mio. Einwohner in Sachsen leben werden (Beyer, 2011; Reiners, 2008).

Die Entwicklung in den neuen Bundesländern unterliegt anderen Pfadabhängigkeiten als in den alten. Der Freistaat hatte nach der Wiedervereinigung im Partnerland BadenWürttemberg seinen Ratgeber gefunden. Der Institutionentransfer aus dem Südwesten hat eine bedeutende Rolle gespielt, weshalb der sächsische Behördenaufbau auf die dortigen Strukturen grundsätzlich rückführbar ist. Hingegen gehen die Entwicklungen in den alten Bundesländern überwiegend auf den Mittelinstanzenbericht zurück, der allerdings auch für die Entscheidungen im Osten eine gewisse Rolle spielt. Grundlegend überdacht wurden die Strukturen in den alten Ländern bereits ab den späten 1960er Jahren. Insbesondere die Oppositionen wollten damals eine Ebene einsparen. 1971 setzte die Ständige Konferenz der Innenminister einen Arbeitskreis ein. Der Bericht von 1973 hatte für die Politik tragende Bedeutung. Er konstatierte u.a. ein hohes Maß an Heterogenität zwischen den Bezirken bezüglich Fläche, Einwohnern, Behördengröße und Zahl der Kommunen. Er empfahl daher Modifikationen hinsichtlich dem Gebiets- und Aufgabenzuschnitt und plädierte für eine Mittelinstanz in größeren Flächenländern ab 3,5 Mio. Einwohnern und einer Fläche ab 20.000 qkm (Mittelinstanzenbericht, 1973; Ellwein, 1994). Die Festlegungen des Mittelinstanzenberichts und die darin explizierten Orientierungswerte haben damit eine Basis für einen Zustand geschaffen, der sich bis heute weitgehend erhalten hat. Der Umstand, dass unter der Voraussetzung bestimmter Konstellationen eine überwiegende Orientierung daran erfolgt, erhebt den Bericht zu einem quasi allgemeingültigen Regelwerk (Reiners, 2008, S. 71-78). 
Mittlerweile vielfach geänderte Rahmenbedingungen dürfen jedoch die durchaus berechtigte Frage zulassen, ob dieser Bericht noch volle Gültigkeit haben kann, ob demnach auch die alten Länder noch in zukunftsfähigen Strukturen arbeiten. Diese haben sich, mit Ausnahme von Niedersachsen, bis heute an die Vorgaben gehalten. Dort wurde 2005 ein radikaler Wandel hin zur Zweistufigkeit vollzogen, was bestimmte Problemfelder, wie die staatliche Repräsentanz und Koordinationsfähigkeit in der Fläche, die Begrenzung der Sonderverwaltung sowie die unterschiedliche Kapazität der kommunalen Kreisstufe, tangiert. Für eine Zweigliedrigkeit ist erforderlich, dass entsprechend personalstarke Ministerien und eine personell gut aufgestellte untere Ebene bestehen und es gerade dort nicht zu Reduktionen kommt (Hennecke, 2004, S. 17; Pickenäcker, 2007, 94; Reiners, 2008; Reiners, 2009, S. 261-279; Hesse 2010, S. 20-21; Hesse, 2011 a, S. 22).

Blickt man im Sinne einer Gesamtschau auf den Verwaltungsumbau in Niedersachsen, so ist zu sehen, dass der dortige Verwaltungsprozess nach anfänglichen Schwierigkeiten wie anderswo funktioniert. Es sind nur wenige Probleme mit einem verkürzten Widerspruchsverfahren erkennbar, Genehmigungsverfahren werden nicht wesentlich verzögert, und anfängliche Ängste sind weitgehend zerstreut worden. Kritische Stimmen und Forderungen sind nach dem Wegfall der Bezirksregierungen dahingehend zu vernehmen, dass nur eine Kreisreform weiterhelfen könne, von Koordinationsschwierigkeiten ist zu hören und vom Klaffen einer Lücke (Bogumil \& Ebinger, 2008; Hellmold, 6.1.2010; Bogumil \& Grohs, 2010). Allerdings ist auch zu betonen, dass fundierte Evaluationen fehlen, die valide Aussagen darüber zulassen, ob dort eher eine Drei- oder eine Zweistufigkeit angemessen wäre. Hesse wertet die Veränderungen in Niedersachsen - wie auch die in Baden-Württemberg - als die in diesem Kontext erfolgreichste Reform. Bei allen Kritikpunkten und der auch kursierenden Auffassung, dass der Wegfall der Mittelinstanz bislang keine signifikanten Unterschiede erbracht hätte, erscheint ein Verzicht auf diese Instanzen nicht nur vertretbar, sondern im Sinne eines modernen Staatsverständnisses durchaus gerechtfertigt. Hinzu kommen Einspareffekte, da Behörden auf der mittleren Ebene kostenwirksame Bürokratisierungstendenzen entfalten und dreistufige Verwaltungssysteme einer diskussionswürdigen Doppelverwaltung Vorschub leisten. Die Leistungsfähigkeit dieses Modells muss sich langfristig aber erst noch empirisch beweisen (2011 b, S. 6, 9-20, 141-158; 2010, S. 2, 23-24, 41, 266; 2011 a, S. 25; 2007; Reiners, 2008).

Die Ausführungen legen nahe, dass ein zweistufiges Modell u.U. wegweisend für Länder sein könnte, die kleiner sind als Niedersachsen. Es ist jedoch erkennbar, dass die niedersächsische Variante keine tragende Bedeutung für die sächsischen Strukturentscheidungen hatte, auch wenn aufgrund der Größenklassifizierung eine Zweistufigkeit durchaus möglich gewesen wäre. Der Freistaat zählt eher zu den mittelgroßen Ländern mit im Vergleich zu Niedersachsen stärker ausgeprägten unteren Verwaltungsstrukturen, die bei einem Wechsel auf eine Zweigliedrigkeit durchaus Kapazitäten zur Über- nahme weiterer Aufgaben mitbringen. Dies zumindest seit dem Jahr 2008, als auf der unteren sächsischen Verwaltungsstufe eine Konzentration erfolgte (Reiners, 2008, S. 123-145).

Für andere Bundesländer ist zu diagnostizieren, dass aus Koordinations- und Steuerungsgesichtspunkten in den ganz großen Ländern Nordrhein-Westfalen und Bayern eine Dreistufigkeit als unbestritten gilt. Für das im Vergleich zu Niedersachsen etwas größere Baden-Württemberg darf dies hingegen nicht mehr uneingeschränkt gelten, denn Niedersachsen hat ein Policy-Window zu einer Zweistufigkeit auch für derartige Größenklassen aufgestoßen. Des Weiteren ist bekannt, dass sowohl die SPD als auch Die Grünen in der neuen badenwürttembergischen Landesregierung eine Zweistufigkeit präferieren und ein passender Umsetzungszeitpunkt lediglich aussteht (Zahariadis, 2008; Reiners, 2008).

Die zu beantwortende Frage lautet demnach weiterhin, welche Momente die sächsische Entscheidung angeleitet haben und beiläufig auch, wie diese zu bewerten ist. Eine Rolle, an einer Dreigliedrigkeit festzuhalten, spielt sicherlich Baden-Württemberg, denn der Südwesten sekundierte nach der Wiedervereinigung beim Aufbau der Strukturen. Blickt man auf die Veränderungen, so gelten als Orientierungsmodell überdies insbesondere die Wegweisungen der beiden kleineren Nachbarländer Sachsen-Anhalt und Thüringen, die ebenfalls dreistufig gegliedert sind, jeweils mit einem zentralen Landesverwaltungsamt (Häusler, 2004, S. 147; Schreiter, 2011; Ragnitz, 2007). Als Vergleichsmaßstab sekundierten aber auch die von der Größe vergleichbaren Länder Hessen und mehr noch Rheinland-Pfalz. Beide sind dreistufig organisiert, was ebenfalls eher für eine sächsische Mittelinstanz spricht. Schaut man jedoch hinter die Kulissen, so könnte sich das Argument ins Gegenteil verkehren, denn beide Länder hätten heute einen zweistufigen Aufbau, wären bestimmte Voraussetzungen noch vor wenigen Jahren anders ausgestaltet gewesen, was nachfolgend deutlich wird (Reiners, 2008, S. 232-233).

Wegen der divergierenden Vorstellungen der Koalitionspartner CDU und FDP bestanden in Hessen 1999 und in den Jahren danach eher geringe Handlungsmöglichkeiten. Wäre es nach der CDU gegangen, so wäre Hessen heute zweigliedrig aufgebaut. Ferner votierten die kommunalen Spitzenverbände für zwei Stufen, da es sich um ein mittelgroßes Land mit relativ starken Kommunalstrukturen handelt, das durchaus ohne Mittelebene und ohne eine Territorialreform auskommen könnte. In Rheinland-Pfalz sprechen die eher schwächeren bzw. als different wahrgenommenen Kommunalstrukturen gegen eine einheitliche Präferenzbildung bei den Spitzenverbänden, was ehemals eine Umgestaltung hin zu zwei Stufen verhindert hat, weil dadurch die Vetopositionen zu ausgeprägt waren. Ferner bestanden Mitte der 1990er Jahre Meinungsverschiedenheiten in der Regierungskoalition. Die Reform zum 1. Januar 2000 beinhaltete schließlich keine Auflösung der Mittelinstanz sondern eine Abkehr vom territorialen Prinzip. Die Regierung ersetzte hierbei die regional ausgerichteten Mittelbehörden durch funktional orientierte Direktionen. Das Direktionsmodell als „Zwittermodell“ war daher nicht von 
vornherein geplant. Es ist in einem langwierigen, inkrementalistischen und partizipativen Prozess entstanden, der aufgrund der vagen Aussagen im Koalitionsvertrag und der Akteursdifferenzen notwendig wurde. Ersichtlich ist, dass solche Antagonismen erhebliche Sonderentwicklungen in Gang setzen können (Bolz, 2000, S. 41; Reiners, 2008, S. 208-217, 231). Die Mechanismen und Prozessabläufe in Hessen und Rheinland-Pfalz sind in der Verwaltungsöffentlichkeit jedoch nicht derart transparent, dass sie bei den Entscheidungen anderer Länder wahrgenommen werden. Wahrgenommen wird nur die Tatsache, dass die beiden Länder dreigliedrig aufgebaut sind.

\subsection{Akteursinteressen und Modernisierungsverlauf}

Im Freistaat Sachsen regiert derzeit eine schwarz-gelbe Regierung. Die CDU stellt im Landtag die stärkste Fraktion mit 58 Abgeordneten, es folgen die Linken mit 29, die SPD und die FDP mit jeweils 14, die Grünen mit neun und die NPD mit acht Sitzen. Insbesondere die stärkste Oppositionsfraktion der Linken votierte gegen das Vorhaben der Regierung und setzte eher auf eine zweistufige Variante. Mehr noch von Interesse sind jedoch die Positionen der Verbände. Die Landesregierung hat im Frühsommer 2011 eine umfassende Anhörung der organisierten Interessenvertreter durchgeführt. Der Prozess ist daher transparent angelegt. Ersichtlich ist, dass die Positionen stark variieren, wobei natürlich niemals alle Akteurspositionen befriedigt werden können. Exemplarisch sollen hier nur einige markante Positionen wichtiger Interessenvertreter thematisiert werden.

Eher skeptisch äußerte sich der Landesbauernverband zu den Maßnahmen auf der mittleren und unteren Verwaltungsstufe, der den Aspekt der Zentralisierung aufgriff und den damit einhergehenden Verlust der Ansprechpartner vor Ort. Weitere kritische Stimmen finden sich u.a. beim Landesbezirk Sachsen, Sachsen-Anhalt, Thüringen, der Dienstleistungsgewerkschaft ver.di, die insbesondere die Standortfragen stark in Zweifel gezogen hat. Ferner kritisiert auch der SSB Beamtenbund, die Tarifunion Sachsen, die Handwerkskammer Dresden und der Bund der Steuerzahler Sachsen das Vorhaben mit ähnlichen Argumenten. Letzterer thematisierte u.a. die Unkalkulierbarkeit und Intransparenz der Kosten und Einsparungen, die zeitliche Dauer der Reform und - mit Verweis auf Niedersachsen - den fehlenden Mut zu einer Zweistufigkeit, was nach deren Ansicht ökonomisch effizient wäre (jeweils 2011; Schreiter, 2011). Daran anknüpfend beklagte das ifo-Institut schon im Jahr 2007 den fehlenden Mut zu effizienteren zwei Stufen (Ragnitz, 2007).

Im Gegensatz hierzu sind auch positive Argumente zu vernehmen. Exemplarisch wird auf zwei wichtige Akteure verwiesen; auf die beiden sächsischen kommunalen Spitzenverbände, deren Interessen bei derartigen Strukturentscheidungen immer nachhaltig berührt sind, weil in deren Machtsphären ganz besonders eingegriffen wird. Der Sächsische Städteund Gemeindetag begrüßte die Reform grundsätzlich, aber auch er kritisierte moderat die Kostenfolgeabschätzung und äußerte Befürchtungen, dass sich künftig Genehmigungsverfahren verzögern werden. Die Sorge tendierte auch dahin, dass es in einigen Gebieten - insbesondere im ländlichen Raum durchaus zu einer Negativentwicklung kommen könnte (2011; Schnerrer, 2011 b). Zu einer Positionierung hinsichtlich einer Zwei- oder Dreistufigkeit gab es beim Städte- und Gemeindetag keine Beschlussfassung. Noch eindeutiger ist die positive Haltung des Landkreistages. Der Spitzenverband positionierte sich klar für eine Dreistufigkeit, aber ebenso für eine bessere Eingliederung von Sonderbehörden wie in BadenWürttemberg, was bislang zu kurz gekommen sei. Der Verband betonte seine Zurückhaltung mit der Begründung, es sei Sache des Staates, wie dieser seine Strukturen organisiert. Er erklärte sich damit quasi solidarisch mit der Regierung. Ferner sprach sich der Landkreistag nachhaltig und, wie noch zu sehen sein wird, aus machtpolitischen Gründen verständlicherweise für eine weitere Kommunalisierung der Aufgaben aus. Moderate Kritikpunkte wurden lediglich hinsichtlich einer Bürgernähe und der „Ausdünnung “ im ländlichen Bereich laut. Der Verband bezeichnete sich diesbezüglich nicht als Gewinner der Reform (2011; Jacob, 2011 a).

Die Maximierung von Bürgernähe zählt heute vielfach zu wichtigen Zielen. Der „Citoyen“ ist heute nicht nur Leistungsempfänger, sondern zählt zum Kunden einer modernen Verwaltung. Entscheidend dürfte in diesem Kontext sein, dass die Maßnahmen auf der mittleren Ebene den Umstand der Bürgernähe weniger tangieren, denn die Mittelinstanzen sind für den Bürger nicht die maßgebliche Anlaufstation. Problematischer sind somit mehr die Standortreduzierungen und Fusionen auf der unteren Stufe, denn Bürgernähe findet vor Ort statt. Eine Vorbedingung für mehr Bürgernähe ist demnach, dass eine Personal- und Standortreduzierung weniger dort oder zumindest mit Augenmaß erfolgt. Weiterhin wurde die untere Stufe erst 2008 gestärkt und konzentriert, ein sicher erheblicher Modernisierungsschritt. Neue Maßnahmen und die Konfrontation der Mitarbeiter mit weiteren Reformen, ohne dass die vorhergehende Reform evaluiert wurde, könnten daher demotivieren. Auf jeden Fall sollte der Prozess den 2008 erst eingeleiteten nicht konterkarieren. Vielfach wird daher betont, dass der vormals eingeschlagene Weg - einer Stärkung der unteren Ebene - der konsequenten Fortführung bedarf (Schreiter, 2011; Jacob, 2011 a; Jacob, 2011 b; Reiners, 2011).

Über die Akteurspositionen hinaus scheint insbesondere der Modernisierungsprozess von Bedeutung. Die Regierung wählte ein transparentes Vorgehen und einen langen Zeitraum. Man kann dies sicherlich als grundsätzlich positives Signal werten (Emminghaus, 2011). Fraglich ist allerdings, ob ein derart langwieriges Vorhaben letztlich auch durchsetzbar ist. Auf alle Fälle dürfte es noch ein weiter Weg werden, die Veränderung letztlich vollständig durchzuführen, denn jede Reform produziert Gewinner und Verlierer. Die Kunst besteht darin, die Summe und das Gewicht der Verlierer möglichst gering zu halten. Ein derart umfassendes Projekt ist mit gewaltigen Unsicherheiten verbunden, die Widerstände wecken. 
Lange Verfahren sind blockadeanfällig. Dies ist sicher keine neue Erkenntnis und es mag auch Ausnahmen geben. Für ähnlich angelegte Strukturreformen, also Modernisierungsmaßnahmen die a priori den Verwaltungsaufbau betreffen, ist dies jedoch empirisch belegt oder zumindest nicht falsifiziert (Reiners, 2011).

Als prominente Beispiele sekundieren die lang anhaltenden Reformversuche fast aller Bundesländer. Vielfach - ist es überhaupt zu einer Reform gekommen - entspricht das Ergebnis dann nicht mehr den ursprünglichen Planungen, wie schon anhand der Länder Hessen oder Rheinland-Pfalz verdeutlicht wurde. Noch anschaulicher wird dies z.B. an den langwierigen Reformbemühungen in Nordrhein-Westfalen, wenngleich dort erschwerende Bedingungen, z.B. vergleichsweise hohe Verwaltungskomplexitäten, hinzukommen. Es wurde schon ausgeführt, dass nur in zwei Ländern, in Niedersachsen und Baden-Württemberg, bislang eine umfassende Strukturreform gelungen ist, jeweils zum 1. Januar 2005. Allerdings waren die Projekte hier nicht auf lange Sicht geplant. Beide Vorhaben bestechen - neben weiteren förderlichen Bedingungen - durch ihre kurze, ja geradezu „machtpolitische“, strategische und radikale Umsetzung, bevor sich überhaupt konzertierter Widerstand regen konnte (Bogumil \& Ebinger, 2005; Reiners, 2008, S. 120-122, 143-145).

\section{Bewertung}

Die lange zeitliche Dauer der Reform erscheint problematisch. Ob eine Umsetzung in einem derart langen Zyklus mit vielen inkrementellen Schritten gelingen wird, muss zumindest zweifelhaft bleiben, und es darf durchaus befürchtet werden, dass nachfolgende Strukturreformen oder andere damit einhergehende Modernisierungsschritte die aktuelle Reform einholen könnten, denn die Halbwertszeiten verkürzen sich heute in allen gesellschaftlichen Bereichen mehr und mehr. Etwas provokant könnte man daher fragen, ob die Reform auf dem längerfristigen Weg zu einer Zweistufigkeit gegebenenfalls nur eine Zwischenlösung darstellt?

Blickt man auf die Modellpräferenz, so wählt Sachsen keinen „radikalen“, riskanteren zweigliedrigen Weg, sondern auch bezogen auf die Dauer der Reform - einen inkrementalistischen. Abermals zeigt sich, dass inkrementalistische Politik eher in das Handlungsfeld von Regierungsparteien fällt, diese daher vielfach eine für mittelgroße und größere Länder traditionelle Dreistufigkeit oder sogenannte „Regierungsmodelle“ verfolgen, weil großartige Einschnitte Ängste wecken und sich mit Widerständen verbinden können. Sie sind somit bestrebt eine Reform schrittweise zu gestalten und sich am Status quo $\mathrm{zu}$ orientieren, weil andernfalls unkalkulierbare politische Kosten produziert werden und ein „non-decision making“ droht (Bachrach \& Baratz, 1963, S. 632-642). Anders als Oppositionsparteien favorisieren Regierungen demnach grundsätzlich keinen radikal-innovativen Systemwechsel. Sie sind sozusagen gezwungen, auf alternative Modelle auszuweichen und eine offensive, diametrale Abgrenzungs- und Differenzierungspolitik zu betreiben. Solche tendieren mehr $\mathrm{zu}$ sogenannten zweistufigen „Oppositionsmodellen“. Ohnehin vollzieht sich der Trend hin zu einer Zweigliedrigkeit vielfach nur über einen Regierungswechsel zwischen den Volksparteien. Der Schritt zu zwei Stufen gelingt eher Oppositionsparteien, die im Zuge der Parteienkonkurrenz für eine Zweigliedrigkeit werben, sich darauf festlegen und durch einen Machtwechsel die Chance bekommen, eine solche auch umzusetzen, sollten weitere förderliche Faktoren und günstige Vetokonstellationen hinzukommen (Reiners, 2008, S. 51).

Weiterhin wurde ausgeführt, dass sich die Vorgänge neoinstitutionalistisch erfassen lassen. Es wurde deutlich, dass die „etablierten“ Modernisierungspfade anderer Länder eine wesentliche Rolle spielen. So orientiert sich Sachsen grundsätzlich an Ländern die eine Dreigliedrigkeit präferieren, die von der Klassifizierung größer oder ähnlich groß sind (z.B. Baden-Württemberg, Hessen oder Rheinland-Pfalz). Die historischen Festlegungen des Mittelinstanzenberichts und die Orientierung vieler Länder an den dort explizierten Klassifizierungen haben bis heute Bestand, wenngleich die Aktualität der Festlegungen mittlerweile berechtigt in Zweifel zu ziehen ist. Der historische Pfad ist im Grundsatz letztlich auf den Institutionentransfer aus dem Südwesten der Republik zurückzuführen, gekoppelt an eine über zwanzigjährige sächsische Emanzipationsphase. Noch entscheidender sind demnach die Wegweisungen der beiden kleineren Nachbarländer Sachsen-Anhalt und Thüringen, die ebenfalls dreigliedrig organisiert sind und darüber hinaus nur eine Bündelungsbehörde aufweisen.

Insgesamt entspringen die kollektiven Positionen der Akteure keiner freien Entscheidung. Sie sind vielmehr interessengeleitet, interaktional vermittelt und institutionell geformt. Dies wird allein an der Position der Fraktion der Linken deutlich. Sie votierte im Zuge der Parteienkonkurrenz gegen das Projekt. Deutlich werden die institutionell geformten Positionen auch an den einzelnen Verbänden. Diejenigen, die für Mitarbeiterinteressen und Rationalisierungsschutz eintreten thematisieren verständlicherweise Standortfragen. Andere betonen stärker ökonomische Kategorien, die Unkalkulierbarkeit der Kosten sowie den langen Reformprozess. Da Politikergebnisse in der Regel über Interaktionen entstehen ist grundsätzlich immer zu erwarten, dass der Weg der Regierung im Zuge einer Verhandlungslösung gegebenenfalls noch modifiziert wird.

Demgegenüber finden sich bei den kommunalen Spitzenverbänden tendenziell positive Argumente, auch wenn diese wichtigen Akteure - aufgrund des dort verständlichen Begehrens nach einer bürgernahen Verwaltung - den Standortentscheidungen auf der unteren Verwaltungsebene eher kritischer begegnen müssten. Dies ist ebenfalls institutionell begründbar. Klar wird die tendenziell positive Position des Städte- und Gemeindetags, wenn man berücksichtigt, dass deren Führungsriege eindeutig christdemokratisch geprägt ist, was vielfach Auswirkungen auf die Meinungsbildung hat (Schnerrer, 2011 a). Noch eindeutiger ist die positive Haltung des Landkreistages, was ebenfalls auf die dortige parteipolitische 
Durchsetzung zurückzuführen ist. Im Landkreistag finden sich nur CDU-Landräte. Die machtpolitischen Verhältnisse zwischen Land und Spitzenverbänden sind somit nicht gegenmajoritär ausgebildet, was den Prozess begünstigt und die Modellpräferenz der Regierung abgesichert hat (Jacob, 2011 a; Reiners, 2008, S. 201).

Im Freistaat zeigt sich, dass die Vetokonstellationen und -positionen institutionell geformt und heterogen ausgebildet sind. Aufgrund ihrer Rationalität finden sich destruktive Vetoplayer, beispielsweise in Gestalt der Gewerkschaften oder des Bundes der Steuerzahler, die in der Lage sind den Prozess institutionell zu blockieren. Es finden sich aber auch potenzielle Vetokräfte, die aufgrund parteipolitischer Determinanten konstruktiv agieren, wie die kommunalen Spitzenverbände. Dies ermöglicht einerseits Entscheidungen, durch die ein problematischer Zustand überwunden werden kann, der zur Veränderung des Status quo beiträgt und zugleich den Willensbildungsprozess für betroffene Interessen und ihre Repräsentanten öffnet (Benz, 2003, S. 205-208).

\section{Konklusion und Ausblick}

Für die Durchsetzung der Reform spricht, dass wesentliche Akteure, die kommunalen Spitzenverbände, das Vorhaben unterstützen. Allerdings sind unter den einzelnen Akteursgruppen auch einige kritische Vetospieler zu finden. Die lange Dauer der Reform wird diese Problematik eher verstärken, denn lange Prozesse schaffen Raum für Diskussionen, Blockade und Stillstand. In der Logik von Politik ist das Zusammenspiel von Rahmenbedingungen und Strategien stets entscheidend. Dieses auf zehn Jahre angelegte strategische Spiel und die Umsetzungspläne werden sich hinsichtlich einer Durchsetzbarkeit empirisch noch beweisen müssen.

Insgesamt machte der Freistaat mit der Reform jedoch einen notwendigen Schritt in die richtige Richtung. Die veränderten finanziellen Rahmenbedingungen und ein verändertes gesellschaftliches Kommunikationsverhalten erforderten effektivere und effizientere Organisationsstrukturen. Die Sächsische Regierung orientierte sich hierbei insbesondere an den beiden Nachbarländern, die ebenfalls eine Dreigliedrigkeit mit nur einer Mittelbehörde präferieren, und die beide von einer Großen Koalition unter CDU-Führung regiert werden. Man kann daher bei einer Dreigliedrigkeit mit nur einer Mittelbehörde durchaus von einem „ostdeutschen dreigliedrigen Modell" sprechen, weil ein solches in den alten Bundesländern nicht vorzufinden ist (Reiners, 2008). Auch hält die Beibehaltung einer Mittelstufe die Widerstände in überschaubareren Grenzen, was die Handlungsmaximen beeinflusst hat. Aus verwaltungswissenschaftlicher Perspektive wäre gegebenenfalls auch eine Zweistufigkeit angezeigt gewesen, denn der Freistaat zählt von der Größenklassifizierung zu den eher mittelgroßen Ländern, mit im Vergleich zum deutlich größeren Niedersachsen doch ansprechend ausgebildeten unteren Verwaltungsstrukturen. Sicherlich darf man die niedersächsischen Rahmenbedingungen nicht unkritisch übertragen. Die entscheidenden Parameter sprechen jedoch durchaus für einen derartigen konsequenten Schritt. Die Empirie zeigt, dass es sich bei zweigliedrigen Modellen, zumindest unterhalb der niedersächsischen Größenklassifizierung, um zukunftsträchtige Varianten handelt. Es handelt sich um Varianten, die angesichts geänderter Rahmenbedingungen vielleicht das künftige Bild der deutschen Verwaltungslandschaft bestimmen könnten.

Diese abschließenden kritischen Bemerkungen erscheinen wichtig, denn die Sächsische Regierung hat im Vorfeld auf einen Politikberatungsprozess gesetzt, der von diversen Akteuren begleitet wurde. Der dabei interessante Aspekt ist sicherlich, was die Politik und Politikberater aus den Prozessen ableiten und was sie gegenseitig voneinander lernen. Politiker können von fundierten wissenschaftlichen Beratungen profitieren, bei denen vielfach auf empirische Ergebnisse zurückgegriffen wird. Dies zumindest aus theoretischer Sicht, denn praktisch bauen politische Mechanismen auf andere Kategorien auf, Kategorien der Macht, des Machterhalts oder des Machterwerbs, was nicht immer mit der vermeintlich besten Lösung kompatibel ist. Findet daher eine Beratungsleistung in einem sehr frühen Stadium statt, in dem noch ergebnisoffen agiert wird, dann besteht durchaus die Möglichkeit inhaltliche Schwerpunkte setzen zu können, wenn auch - konkret auf Verwaltungsstrukturmaßnahmen bezogen - u.U. nur marginal, denn die Modellpräferenzen entspringen ganz bestimmten Maßgeblichkeiten, wie gezeigt wurde. Findet Politikberatung hingegen - wie hier - in einem Stadium statt, in dem sich die Akteure bereits festgelegt haben, so hat die Beratungsleistung für die Politikakteure vielfach lediglich „legitimatorischen Charakter".

\section{Literatur}

Bachrach, P. \& Baratz, M. S. (1963). Decisions and Nondecisions: An Analytical Framework. American Political Science Review, 57(3), 632-642.

Bandelow, N. C. (2003 a). Kollektive Lernprozesse durch Vetospieler? Bochum, Univ., Habil.

Bandelow, N. C. (2003 b). Institutionelle Vetopunkte und kollektive Lernprozesse in der britischen und deutschen Europapolitik der 1980er und 1990er Jahre. In U. Lehmkuhl, H. Zimmermann \& C. Wurm (Hg.), Deutschland, Großbritannien, Nordamerika (S. 91-110). Tübingen: Steiner.

Becker, B. (1989). Öffentliche Verwaltung in Deutschland. Percha: R. S. Schulz.

Benz, A. (2001). Der moderne Staat. München/Wien: Oldenbourg.

Benz, A. (2003). Konstruktive Vetospieler in Mehrebenensystemen. In R. Mayntz \& W. Streek (Hg.), Die Reformierbarkeit der Demokratie (S. 205-236). Frankfurt a.M.: Campus.

Beyer, U. (2011). Sachverständigenanhörung im Sächsischen Landtag: Stenografisches Protokoll (Sächsisches Staatsministeriums des Innern). 26. Sitzung des Verfassungs-, Rechts- und Europaausschusses am 26.9.2011 zum Sächsischen Standortegesetz, Gesetzesentwurf der Regierung, Drs. 5/6429, 7.10.2011.

Bogumil, J. \& Ebinger, F. (2005). Die Große Verwaltungsstrukturreform in Baden-Württemberg. Gutachten. Münster: Stiftung und Verein Westfalen-Initiative.

Bogumil, J. \& Ebinger, F. (2008). Grenzen der Subsidiarität: Verwaltungsreform und Kommunalisierung in den Ländern. In H. Heinelt \& A. Vetter (Hg.), Lokale Politikforschung heute (S. 165-196). Wiesbaden: VSVerlag. 
Bogumil, J. \& Grohs, S. (2010). Möglichkeiten und Grenzen von Regionalverwaltungen. In J. Bogumil \& S. Kuhlmann (Hg.), Kommunale und regionale Aufgabenwahrnehmung im Wandel (S. 89-110). Wiesbaden: Springer VS.

Bolz, H. (2000). Das Direktionsmodell in Rheinland-Pfalz. In G. Konzendorf (Hg.), Neuorganisation der Mittelinstanzen (S. 41-52). Speyerer Forschungsberichte 210. Speyer: Forschungsinstitut für öffentliche Verwaltung bei der Deutschen Hochschule für Verwaltungswissenschaft.

Bouckaert, G. (2004). Die Dynamik von Verwaltungsreformen. In W. Jann, J. Bogumil, G. Bouckaert, L. Holtkamp, L. Kißler, S. Kuhlmann, E. Mezger, C. Reichard \& H. Wollmann (Hg.), Status-Report Verwaltungsreform (S. 22-35). Berlin: edition sigma.

Bund der Steuerzahler Sachsen e.V. (2011). Schriftliche Anhörung zum Gesetzesentwurf der Staatsregierung, Drs. 5/6426, 22.6.2011.

Ellwein, T.(1994). Das Dilemma der Verwaltung. Mannheim/Leipzig/ Wien/Zürich: BI Taschenbuchverlag.

Emminghaus, C. (2011). Sachverständigenanhörung im Sächsischen Landtag: Stenografisches Protokoll (Sächsischer Landkreistag). 26. Sitzung des Verfassungs-, Rechts- und Europaausschusses am 26.9.2011 zum Sächsischen Standortegesetz, Gesetzesentwurf der Regierung, Drs. 5/6429, 7.10.2011.

Freudenberg, D. (2000). Verwaltungsreform im Spannungsfeld von politischer Steuerung und Dezentralisierung. In L. Kißler, N. Kersting \& H. J. Lange (Hg.), Politische Steuerung und Reform der Landesverwaltung (S. 39-52). Baden-Baden: Nomos.

Gisler, M. (2001). Einführung in die Begriffswelt des eGovernment. In M. Gisler \& D. Spahni (Hg.), eGovernment (S. 13-30). Bern: Haupt.

Grabow, B., Siegfried, C. \& Wulff, M. (2006). Kommunales E-Government. Berlin: Deutsches Institut für Urbanistik.

Häusler, B. (2004). Zur Abschaffung der Bezirksregierungen. Niedersächsische Verwaltungsblätter, 11(6), 145-149.

Hall, P. A. \& Taylor, R. C. R. (1996). Political Science and the Three New Institutionalisms. Köln: MPIfG Discussion Paper 6.

Handwerkskammer Dresden (2011). Schriftliche Anhörung zum Gesetzesentwurf der Staatsregierung, Drs. 5/6426, 22.6.2011.

Hellmold, T. (6.1.2010). Ein Nachwort das es in sich hat: Ex-Landtagsdirektor Janssen hält Verwaltungsreform für nutzlos (NWZ-Thema Regionalpolitik). Abgerufen am 8.2.2013, http://www.nwzonline.de.

Hennecke, H. J. (2004). Staat und Verwaltungsreform in Deutschland: Ein Problemaufriss. In H. J. Hennecke (Hg.), Staats- und Verwaltungsmodernisierung in Mecklenburg-Vorpommern: Anregungen, Standpunkte und Perspektiven zur Reformdebatte (S. 7-21). Rostock: Rostocker Informationen zu Politik und Verwaltung, 21.

Hesse, J. J. (2007). Was soll und kann Verwaltungsreform? Der Fall Niedersachsen. Niedersächsische Verwaltungsblätter, 14(6), 145-160.

Hesse, J. J. (2010 a). Kommunalstrukturen in Niedersachsen. Untersuchung im Auftrag des Ministeriums für Inneres und Sport des Landes Niedersachsen. Berlin: Internationales Institut für Staat und Europawissenschaften.

Hesse, J. J. (2011 a). Kommunalstrukturen in Niedersachsen: Eine teilregionale Untersuchung für den Raum Wolfsburg-Gifhorn-Helmstedt. Gutachten im Auftrag der Stadt Wolfsburg und der Landkreise Gifhorn und Helmstedt. Berlin: Internationales Institut für Staats- und Europawissenschaften.

Hesse, J. J. (2011 b). Der Staat in der Fläche: Landesverwaltung ohne Mittelinstanz? Baden-Baden: Nomos.

Jacob, A. (2011 a). Sachverständigenanhörung im Sächsischen Landtag: Stenografisches Protokoll (Sächsischer Landkreistag). 26. Sitzung des Verfassungs-, Rechts- und Europaausschusses am 26.9.2011 zum Sächsischen Standortegesetz, Gesetzesentwurf der Regierung, Drs. 5/6429, 7.10.2011.

Jacob, A. (2011 b). Expertenbefragung im Sächsischen Landtag. 26. Sitzung des Verfassungs-, Rechts- und Europaausschusses am 26.9.2011 zum Gesetzesentwurf der Landesregierung, Drs. 5/6429, 26.9.2011.

Kaiser, A. (1998). Vetopunkte der Demokratie. Zeitschrift für Parlamentsfragen, 29(3), 524-541.

Kaiser, A. (1999). Die politische Theorie des Neo-Institutionalismus. In A. Brodocz \& G. S. Schaal (Hg.), Politische Theorien der Gegenwart (S. 189-211). Opladen: Leske + Budrich).
Lucke, J. v. \& Reinermann, H. (2000). Speyerer Definition von E-Government. Speyer: Forschungsinstitut für öffentliche Verwaltung bei der Deutschen Hochschule für Verwaltungswissenschaft.

Lüder, K. (2004). Zur Erklärung von Reformprozessen in Verwaltungen. In A. Benz, H. Siedentopf \& K.-P. Sommermann (Hg.), Institutionenwandel in Regierung und Verwaltung (S. 75-88). Berlin: Duncker \& Humblot.

Luhmann, N. (1984). Soziale Systeme. Frankfurt a.M.: Suhrkamp.

Mayntz, R. \& Scharpf, F. W. (1995). Der Ansatz des akteurszentrierten Institutionalismus. In R. Mayntz \& F. W. Scharpf (Hg.), Gesellschaftliche Selbstregelung und politische Steuerung (S. 39-72). Frankfurt a.M.: Campus.

Mittelinstanzenbericht (1973). Neuordnung der staatlichen Mittelinstanzen (vorgelegt vom Sonderarbeitskreis der Ständigen Konferenz der Innenminister der Länder).

Nemitz, C. (2000). Erfolgsfaktoren für eine Reform politischer Systeme. Speyerer Forschungsberichte 208. Speyer: Forschungsinstitut für öffentliche Verwaltung bei der Deutschen Hochschule für Verwaltungswissenschaft.

Pickenäcker, B. A. (2007). Bezirksregierungen in NRW: Entwicklung Transformation - Perspektive (Schriftenreihe der Stiftung Westfalen-Initiative, Bd. 12). Münster.

Ragnitz, J. (2007). Überlegungen zur geplanten Verwaltungsstrukturreform im Freistaat Sachsen. Im Blickpunkt, 5/2007, 45-48. Abgerufen am 7.3.2013, http://www.cesifo-group.de.

Reiners, M. (2008). Verwaltungsstrukturreformen in den deutschen Bundesländern: Radikale Reformen auf der Ebene der staatlichen Mittelinstanz. Wiesbaden: VS-Verlag.

Reiners, M. (2009). Moderne Staatsarchitektur und tief greifende Reformen in Niedersachsen. Verwaltungsarchiv, 100(2), 261-279.

Reiners, M. (2011). Sachverständigenanhörung im Sächsischen Landtag: Stenografisches Protokoll. 26. Sitzung des Verfassungs-, Rechts- und Europaausschusses am 26.9.2011 zum Sächsischen Standortegesetz, Gesetzesentwurf der Regierung. Drs. 5/6429, 7.10.2011.

Sächsische Staatsregierung (2011). Gesetzesentwurf: Gesetz zur Neuordnung von Standorten der Verwaltung und der Justiz des Freistaates Sachsen (Sächsisches Standortegesetz - SächsStOG), Vorblatt (1-2) und Übersicht über die Auswirkungen der Vorlage, Drs. 5/6426, Stand Juli 2011.

Sächsischer Landesbauernverband e.V. (2011). Schriftliche Anhörung zum Gesetzesentwurf der Staatsregierung, Drs. 5/6426, 22.6.2011.

Sächsischer Landkreistag (2011). Schriftliche Anhörung zum Gesetzesentwurf der Staatsregierung, Drs. 5/6426, 22.6.2011.

Sächsischer Städte- und Gemeindetag (2011). Schriftliche Anhörung zum Gesetzesentwurf der Staatsregierung, Drs. 5/6426, 22.6.2011.

Scharpf, F. W. (2000). Interaktionsformen: Akteurszentrierter Institutionalismus in der Politikforschung. Opladen: UTB.

Scheer, A. W., Heib, R. \& Kruppke, H. (2003). E-Government. Berlin/ Heidelberg: Springer.

Schnerrer, C. (2011 a). Expertenbefragung im Sächsischen Landtag. 26. Sitzung des Verfassungs-, Rechts- und Europaausschusses am 26.9.2011 zum Gesetzesentwurf der Landesregierung, Drs. 5/6429, 26.9.2011.

Schnerrer, C. (2011 b). Sachverständigenanhörung im Sächsischen Landtag: Stenografisches Protokoll (Sächsischer Städte- und Gemeindetag). 26. Sitzung des Verfassungs-, Rechts- und Europaausschusses am 26.9.2011 zum Sächsischen Standortegesetz, Gesetzesentwurf der Regierung, Drs. 5/6429, 7.10.2011.

Schreiter, K. (2011). Sachverständigenanhörung im Sächsischen Landtag: Stenografisches Protokoll (Bund der Steuerzahler Sachsen e.V.). 26. Sitzung des Verfassungs-, Rechts- und Europaausschusses am 26.9.2011 zum Sächsischen Standortegesetz, Gesetzesentwurf der Regierung, Drs. 5/6429, 7.10.2011

Seitz, H. (2007). Fiskalische und ökonomische Effekte der Verwaltungsreform in Sachsen. Dresden: ohne Verlag.

Siedentopf, H. \& Laux, E. (1998). Funktionalreform in Sachsen. BadenBaden: Nomos.

SSB Beamtenbund und Tarifunion Sachsen (2011). Schriftliche Anhörung zum Gesetzesentwurf der Staatsregierung, Drs. 5/6426, 22.6.2011. 
Statistisches Bundesamt (2011). Einwohnerzablen (Stand 31.12.2011). Abgerufen am 20.12.2012, http://www.statistik-portal.de.

Steinmo, S., Thelen, K. \& Longstreth, F. (Hg.). (1992). Structuring Politics: Historical Institutionalism in Comparative Analysis. Cambridge: Cambridge University Press.

Thelen, K. (2002). How Institutions Evolve: Insights from ComparativeHistorical Analysis. In J. Mahoney \& D. Rueschemeyer (Eds.), Comparative Historical Analysis in the Social Science (S. 208-240). New York: Cambridge University Press.

Evans, P. B., Rueschemeyer, D. \& Skocpol, T. (Hg.). (1985). Bringing the State Back In. Cambridge: Cambridge University Press.

Tsebelis, G. (1995). Decision Making in Political Systems: Veto Players in Presidentialism, Parliamentarism, Mulitcameralism and Multipartyism. British Journal of Political Science, 25(3), 289-325.

Tsebelis, G. (2002). Veto Players: How Political Institutions Work. New York: Sage.

Ver.di, Vereinigte Dienstleistungsgewerkschaft, Landesbezirk Sachsen, Sachsen-Anhalt, Thüringen (2011). Schriftliche Anhörung zum Gesetzesentwurf der Staatsregierung, Drs. 5/6426, 22.6.2011.

Wirtschaftswoche (22.10.2011). Initiative Nene Soziale Marktwirtschaft (Länder-Ranking 2010).
Zahariadis, N. (2008). Ambiguity and choice in European public policy. Journal of European Public Policy, 15(4), 514-530.

ZEIT Online (27.5.2004). E-Mail ans Amt. Abgerufen am 6.5.2013, http://www.zeit.de.

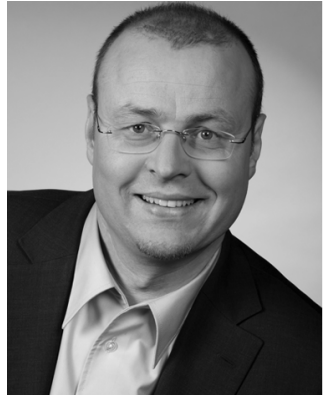

Dr. phil. Markus Reiners ist Lehrbeauftragter (Post-Doc) an der Leibniz Universität Hannover. Er studierte Politikwissenschaft, Psychologie und Rechtswissenschaften in Hagen. Neben seiner Lehr- und Forschungstätigkeit engagiert er sich in der wissenschaftlichen Politikberatung. Email: markus.reiners@tonline.de

\title{
Willy Brandt: Inszenierung des deutschen Kennedys
}

\author{
Johannes Pütz
}

\section{Kernaussagen}

Politische Akteure werden öffentlich konstruiert und inszeniert. Am Beispiel Willy Brandts in seiner Zeit als Regierender Bürgermeister Berlins werden in diesem Beitrag die Strategien der Konstruktion eines Images analysiert. Die öffentliche Person Willy Brandt galt als Ikone für die Freiheit und Bollwerk gegen den Kommunismus. Gezielt wird auf private Darstellungen gesetzt, um ihn als politischen Akteur in der Rolle eines jungen Helden zu zeigen: des „deutschen Kennedy“.

\section{Einleitung}

Die Darstellung emotionaler Befindlichkeiten und privater Verhältnisse von politischen Akteuren gerät zunehmend in den Blickpunkt der medialen Berichterstattung (Donk \& Westerbarkey, 2009, S. 32). Die mediale Inszenierung des Politischen, der jede politische Darstellung unterliegt, wirft dabei die Frage nach der „Substanz des Politischen in der medialen Darstellungsform “ auf (Classen \& Mergel, 2007, S. 53). Denken wir an den Auftritt des ,Medienkanzler' Gerhard Schröder, so ist das medial inszenierte Politikerimage des Brioni tragenden Dressmans mit Cohiba im Kopfkino präsent (vgl. Meng, 2002; Rosumek, 2007). Merkels Darstellung im dekolletierten Kleid in der Osloer Oper belegt diesen Trend. Politische Kommunikation reagiert strategiebasiert auf diese Tendenzen und Politiker bedienen sich ihrer Medien- und Kom- munikationsberater, um ein positives Image in den Medien zu erzeugen.

Auch Willy Brandt genoss den Status eines internationalen Medienstars. Und das schon vor seiner Kanzlerschaft. Darstellungen Brandts in der Ufa-Wochenschau sind bisher nicht untersucht worden. Auch Zeitschriftenaufsätze liefern wenig zur medialen Inszenierung Brandts (vgl. Rosumek, 2007, S. 100). Dieser Beitrag analysiert die mediale Inszenierung Willy Brandts in der Ufa-Wochenschau von 1957 bis 1964 und beschäftigt sich mit Darstellungsstrategien in den UfaSujets, mittels derer die ,Ikone Brandt' (Stolzmann, 2005) in den Rollen und Images als Berliner Aufsteiger, „deutscher Kennedy“1 (Münkel, 2003, S. 60), „Staatsmann im Werden“ (Merseburger, 2002, S. 350) und „der erste und einzige politische Superstar der Bundesrepublik“ (Münkel, 2005, S. 291) ein Star-Framing ${ }^{2}$ erfährt. Die empirische Grundlage bildet ein Sample von acht Ufa-Beiträgen über Brandt als Ikone des 20. Jahrhunderts, deren Darstellung mit der Wahl zum Regierenden Bürgermeister im Oktober 1957 beginnt: „The flamboyant Willy Brandt, the first SPD chancellor of West Germany, intially became a national figure while serving as mayor of West Berlin“ (Davis, 1998, S. 107). Gilt Willy Brandt ferner

1 Ich danke Daniel März für zahlreiche Tipps und Hinweise. Die Ufa 692 kommentiert: „Ein wenig Deutschlands Kennedy“ (Time Code Reading: TCR 10:01:28).

2 Die Begriffe Rahmung, Framing und Deutungsmuster werden in diesem Beitrag synonym verwendet. Die Auswahl des Frames strukturiert die Meinungsbildung zu einem Thema. In Anlehnung an Mannheim lassen sich Frames als „Tiefenstrukturen öffentlicher Meinung“ klassifizieren (Eilders, 2008, S. 41). 